Research Article

\title{
Forgotten Coindex for the Derived Sum Graphs under Cartesian Product
}

\author{
Muhammad Ibraheem, ${ }^{1}$ Meshari M. Aljohani, ${ }^{2}$ Muhammad Javaid $\left(D,{ }^{1}\right.$ \\ and Abdulaziz Mohammed Alanazi $\mathbb{1}^{3}$ \\ ${ }^{1}$ Department of Mathematics, School of Science, University of Management and Technology, Lahore 54770, Pakistan \\ ${ }^{2}$ Department of Chemistry, University of Tabuk, Tabuk, Saudi Arabia \\ ${ }^{3}$ Department of Mathematics, University of Tabuk, Tabuk, Saudi Arabia
}

Correspondence should be addressed to Muhammad Javaid; javaidmath@gmail.com

Received 29 July 2021; Accepted 18 September 2021; Published 4 October 2021

Academic Editor: Syed Ahtsham Haq Bokhary

Copyright (C) 2021 Muhammad Ibraheem et al. This is an open access article distributed under the Creative Commons Attribution License, which permits unrestricted use, distribution, and reproduction in any medium, provided the original work is properly cited.

\begin{abstract}
A topological index (TI) is a molecular descriptor that is applied on a chemical structure to compute the associated numerical value which measures volume, density, boiling point, melting point, surface tension, or solubility of this structure. It is an efficient mathematical method in avoiding laboratory experiments and time-consuming. The forgotten coindex of a structure or (molecular) graph $H$ is defined as the sum of the degrees of all the possible pairs of nonadjacent vertices in $H$. For $D \in\{S, R, Q, T\}$ and the connected graph $H$, the derived graphs $D(H)$ are obtained by applying the operations $S$ (subdivided), $R$ (triangle parallel), $Q$ (line superposition), and $T$ (total graph), respectively. Moreover, a derived sum graph ( $D$-sum graph) is obtained by the Cartesian product of the graph $H_{2}$ with the graph $D\left(H_{1}\right)$. In this study, we compute forgotten coindex of the $D$-sum graphs $H_{1+S} H_{2}$ (S-sum), $H_{1+R} H_{2}$ (R-sum), $H_{1+Q} H_{2}\left(Q\right.$-sum), and $H_{1+T} H_{2}$ (T-sum) in the form of various indices and coindices of the factor graphs $\mathrm{H}_{1}$ and $\mathrm{H}_{2}$. At the end, we have analyzed our results using numerical tables and graphical behaviour for some particular $D$-sum graphs.
\end{abstract}

\section{Introduction}

Chemical graph theory being the combination of graph theory and chemistry is a branch of mathematical chemistry in which we study the various physical and chemical properties of a chemical structure or network using different graph theoretical techniques. A topological index is one of the most used graph theoretical technique that studies the different properties of the molecular structure such as volume, density, freezing point, vaporization point, boiling point, melting point, surface tension or solubility, heat of formation, and heat of evaporation numerically $[1,2]$.

TIs are categorized in three types such as degree, distance, and polynomial based, but according the latest survey [3], the degree-based TIs are more studied than others. Wiener was the first scientist who calculated the boiling point of paraffin by using a distance-based TI [4]. Gutman and Trinajsti [5] introduced degree-based TIs known as
Zagreb indices to calculate total $\pi$-electron energy of hydrocarbons. Rouvary $[6]$ and Balaban $[7,8]$ discussed the structure-activity correlations of different chemical phenomenon using TIs. Klein et al. introduced a molecular topological index and drive a close relation with the Wiener index $[9,10]$. Mendiratta et al. and Cornwell used Wiener's TI to study structure-activity study on antiviral 5-vinylpyrimidine nucleoside analogs [11]. Gutman and Estrada calculated the TIs based on the line graph of the molecular graph [12]. Biye et al. wrote a novel on TI for QSPR/QSAR study of organic compounds [13]. Qinghua et al. calculated the TI for octane number of acyclic alkane by autocorrelation [14]. Baig et al. computed the TIs for polyoxide and silicate DSL and DOX-like networks [15].

In 2015, Furtula and Gutman redefined a TI with the name forgotten index ( $F$-index) as the sum of cubes of vertex degrees of a molecular graph [16]. Gutman et al. listed the graphs having smallest forgotten index [17]. Zhongyuan and 
Zhibo computed bounds of the F-index using CauchySchwarz inequality, Jensen's inequality, and Chebyshev's sum inequality [18]. Gao and Liu calculated $F$-index of different chemical structures $[19,20]$. Ahmad et al. worked online graph of benzene ring in the 2D network and calculated the degree-based TIs for these graphs [21]. Javaid et al. calculated bound on $F$-index for unicyclic graphs with fixed number of pendent vertices [22]. Imran et al. [23] investigated the family of unicyclic graphs with extreme $F$-coindex.

Ashrafi et al. introduced the concept of coindex of graph and investigated Zagreb coindices of composite graph operations such as union, disjunction, and various product of graphs [24]. Havare et al. computed the F-index and $F$-coindex for carbon base nanomaterial [25]. Basavanagoud and Desai calculated the $F$-index and hyper-Zagreb index of generalized transformation graphs [26]. Gao et al. calculated electron energy of molecular structures $F$-index [27]. Yasir et al. computed $F$-index of dendrimers-like structure [28]. Basavanagoud and Timmanaikar calculated first Zagreb and $F$-index of some dominating transformation graphs [29]. Sana et al. characterized the extremal graphs and proved the ordering among the different subfamilies of graphs with respect to $F$-index [30].

Various operations on a graph play the important role in the development of different new classes of graphs. Yan et al. listed five graphs line graph $L(H)$, subdivided graph $S(H)$, line superposition graph $Q(H)$, triangle parallel $R(H)$, and total $T(H)$ by performing different operations on $H$ and computed Wiener indices of these five graphs [31]. Eliasi and Taeri defined the derived sum graphs $\left(H_{1+D} H_{2}\right)$ and computed their Wiener indices, where $D \in\{S, R, Q, T\}$ [32]. Later on, for these derived sum graphs, Deng et al. [33] computed first two Zagreb indices, and Akhtar and Imran calculated the $F$-index [34, 35]. Javaid et al. (2021) investigated the first Zagreb connection index and coindex [36], and Javaid et al. investigated forgotten index of these graphs [37]. Moreover, Pattabiraman and Peng computed $F$-indices and their coindices of some classes of graphs $[38,39]$. Ali et al. forgotten coindex of some nontoxic dendrimers structure used in targeted drug delivery [40].

In this study, we compute forgotten coindices of $D$-sum graphs $H_{1+D} H_{2}$, where $D \in\{S, R, Q, T\}$ in the form of forgotten index, first Zagreb indices, and coincides of their basic graphs $H_{1}$ and $H_{2}$. At the end, the obtained results are also illustrated with the assistance of the examples for some particular $D$-sum graphs. In Section 2, the definitions and notations are presented, Section 3 includes the main results of our work, and Section 4 presents particular examples related to the main results.

\section{Preliminaries}

Let $H=(V(H), E(H))$ be a connected graph, where $V(H)$ and $E(H)$ be the set of vertices and edges of $H$, respectively. For any vertex $x \in V(H)$, its degree is denoted by $d(x)$ and defined as the number of edges connecting it. The joining of two vertices $x, y \in V(H)$ formed an edge denoted by $x y \in E(H)$. Gutman and Trinajstic [5] introduced Zagreb indices $M_{1}(H)$ and $M_{2}(H)$ to calculate total $\pi$ electron energy of hydrocarbons. The Zagreb indices $M_{1}(H)$ and $M_{2}(H)$ are defined as

$$
\begin{aligned}
& M_{1}(H)=\sum_{r_{1} r_{2} \in E(H)}\left[d_{H}\left(r_{1}\right)+d_{H}\left(r_{2}\right)\right], \\
& M_{2}(H)=\sum_{r_{1} r_{2} \in E(H)}\left[d_{H}\left(r_{1}\right) d_{H}\left(r_{2}\right)\right] .
\end{aligned}
$$

Ashrafi et al. [24] defined Zagreb coincides such as $\bar{M}_{1}(H)$ and $\bar{M}_{2}(H)$ of the Zagreb indices. The Zagreb coindices are defined as

$$
\begin{aligned}
& \bar{M}_{1}(H)=\sum_{r_{1} r_{2} \notin E(H)}\left[d_{H}\left(r_{1}\right)+d_{H}\left(r_{2}\right)\right], \\
& \bar{M}_{2}(H)=\sum_{r_{1} r_{2} \notin E(H)}\left[d_{H}\left(r_{1}\right) d_{H}\left(r_{2}\right)\right] .
\end{aligned}
$$

Furtula and Gutman [16] defined forgotten index ( $F$-index), and its mathematical form is given by

$$
F(H)=\sum_{r_{1} r_{2} \in E(H)}\left[d_{H}\left(r_{1}\right)^{2}+d_{H}\left(r_{2}\right)^{2}\right] .
$$

Nilanjan et al. [41] introduced the forgotten coindex ( $F$-coindex) for $F$-index with mathematical formulation as

$$
\bar{F}(H)=\sum_{r_{1} r_{2} \notin E(H)}\left[d_{H}\left(r_{1}\right)^{2}+d_{H}\left(r_{2}\right)^{2}\right] .
$$

Let $H$ be a simple connected graph; then, its derived graphs are defined as follows.

(i) $S(H)$ is a graph formed by inserting a new vertex in each edge of $H$

(ii) $R(H)$ is a graph obtained from $S(H)$ by adding an edge between the adjacent vertices of $H$

(iii) $Q(H)$ is a graph formed from $S(H)$ by adding an edge between the pairs of new vertices which are on the adjacent edges of $H$

(iv) $T(H)$ is formed by performing both operations of $R(H)$ and $Q(H)$ on $S(H)$

Suppose that $H_{1}$ and $H_{2}$ are two connected graphs; then, their derived sum graph ( $D$-sum graphs) is denoted by $H_{1+D} H_{2}$ and defined with vertex set $V\left(H_{1+D} H_{2}\right)=$ $V\left(H_{1}\right) \cup E\left(H_{1}\right) \times V\left(H_{2}\right)$, and edge set is defined as the vertices $\left(r_{1}, r_{2}\right)$ and $\left(s_{1}, s_{2}\right)$ of $H_{1+D} H_{2}$, where $D \in\{S$, $R, Q, T\}$ are joined iff.

$$
\begin{aligned}
& \text { (i) } r_{1}=s_{1} \in V\left(H_{1}\right) \text { and } r_{2} \backsim s_{2} \in H_{2} \\
& \text { (ii) } r_{2}=s_{2} \in V\left(H_{2}\right) \text { and } r_{1} \backsim s_{1} \in D\left(H_{1}\right)
\end{aligned}
$$

Where $r \backsim s \in H_{2}$ presents the $x y$ is an edge in $H_{2}$ [26]. For the $D$-sum graphs, refer Figures 1 and 2.

\section{Main Results}

In this section, we discuss main results related to $F$-coindex for $D$-sum graphs. Let $H_{1}$ be a graph of its order, and the size is denoted by $n_{1}$ and $e_{1}$, respectively. Let $\overline{H_{1}}$ be the complement of $H_{1}$, and the edge set for $\overline{H_{1}}$ is given by 

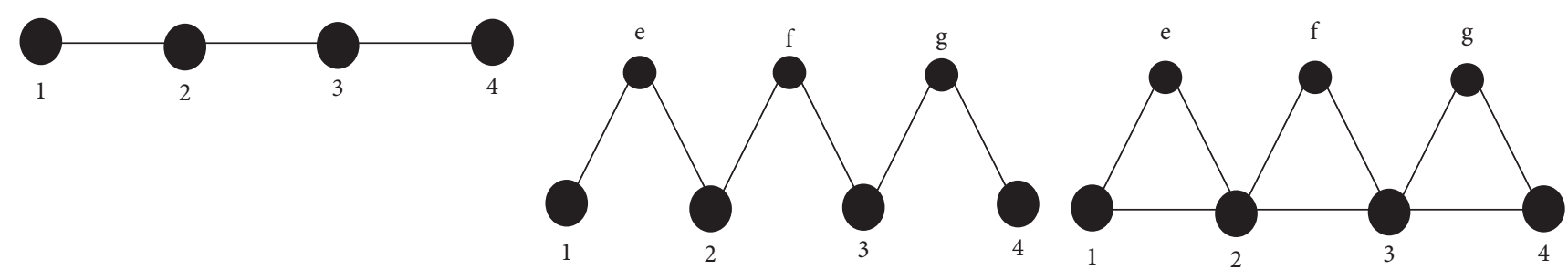

(a)

(b)

(c)

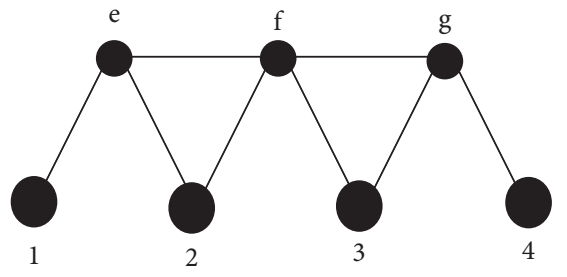

(d)

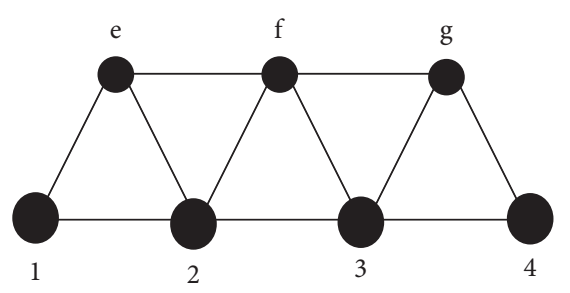

(e)

Figure 1: (a) $G \cong P_{4}$, (b) $S(G) \cong S\left(P_{4}\right)$, (c) $R(G) \cong R\left(P_{4}\right)$, (d) $Q(G) \cong Q\left(P_{4}\right)$, and (e) $T(G) \cong T\left(P_{4}\right)$.
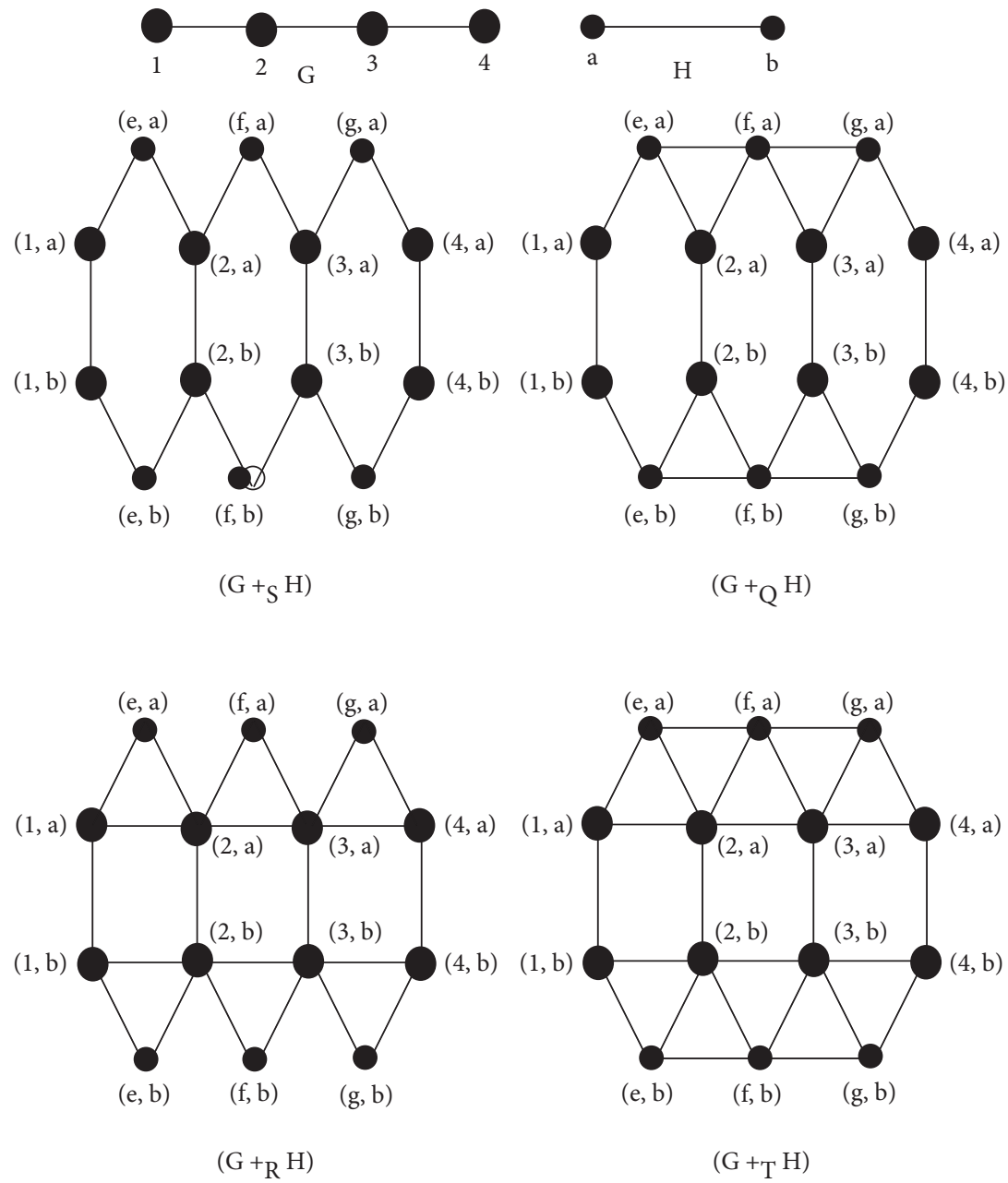

Figure $2: G \cong P_{4}, H \cong P_{2}$, and $G_{+D} H \cong P_{4+D} P_{2}$. 
$\left(\begin{array}{c}n_{1} \\ 2\end{array}\right)-e_{1}$. Further assumed some sums by $\alpha, \alpha_{1}, \alpha_{2}, \alpha_{3}$, and $\alpha_{4}$ are given as follows.

(i) $\alpha=\left(n_{2}\left(n_{2}-1\right)+\bar{e}_{2}\left(\bar{n}_{2}-1\right)\right)$

(ii) $\left.\alpha_{1}=\sum_{\substack{r_{1} r_{2} \notin E\left(D\left(H_{1}\right)\right) \\ r_{1} \in V\left(H_{1}\right) \\ r_{2} \in V\left(D\left(H_{1}\right)-V\left(H_{1}\right)\right)}}\left[d_{H_{1}}\left(r_{1}\right)^{2}+d_{D\left(H_{1}\right)}\left(r_{2}\right)^{2}\right)\right]$

(iii) $\left.\alpha_{2}=\sum r_{1} r_{2} \notin E\left(D\left(H_{1}\right)\right)\left[d_{H_{1}}\left(r_{1}\right)^{2}+d_{D\left(H_{1}\right)}\left(r_{2}\right)^{2}\right)\right]$ $r_{1} \in V\left(H_{1}\right)$
$r_{2} \in V\left(D\left(H_{1}\right)-V\left(H_{1}\right)\right)$

$$
\begin{gathered}
\text { (iv) } \alpha_{3}=\sum_{\substack{r_{1} r_{2} \notin E\left(D\left(H_{1}\right)\right) \\
r_{1} \in V\left(H_{1}\right) \\
r_{2} \in V\left(D\left(H_{1}\right)-V\left(H_{1}\right)\right)}} d_{H_{1}}\left(r_{1}\right) \\
\text { (v) } \alpha_{4}=\sum_{\substack{r_{1} r_{2} \notin E\left(D\left(H_{1}\right)\right) \\
r_{1} \in V\left(H_{1}\right) \\
r_{2} \in V\left(D\left(H_{1}\right)-V\left(H_{1}\right)\right)}} d_{H_{1}}\left(r_{1}\right)
\end{gathered}
$$

Theorem 1. Let $H_{1+S} H_{2}$ be a S-sum graph; then, its F-coindex is

$$
\begin{aligned}
\bar{F}\left(H_{1+S} H_{2}\right)= & 4\left(n_{2}^{2} e_{1}^{2}-n_{2} e_{1}\right)+2 \bar{e}_{2} M_{1}\left(H_{1}\right)+4 e_{1} \bar{M}_{1}\left(H_{2}\right)+n_{1} \bar{F}\left(H_{2}\right)+n_{2}\left(F\left(H_{1}\right)+\bar{F}\left(H_{1}\right)\right) \\
& +2\left(e_{1}+\bar{e}_{1}\right)+\left(e_{1}\left(n_{1}-2\right)\right) M_{1}\left(H_{2}\right)+4 e_{2}\left(M_{1}\left(H_{1}\right)+\bar{M}_{1}\left(H_{1}\right)\right)+2\left[( e _ { 2 } + \overline { e } _ { 2 } ) \left(F\left(H_{1}\right)\right.\right. \\
& \left.\left.+\bar{F}\left(H_{1}\right)\right)+\left(e_{1}+\bar{e}_{1}\right)\left(F\left(H_{2}\right)+\bar{F}\left(H_{2}\right)\right)+\left(M_{1}\left(H_{2}\right)+\bar{M}_{1}\left(H_{2}\right)\right)\left(M_{1}\left(H_{1}\right)+\bar{M}_{1}\left(H_{1}\right)\right)\right] \\
& +n_{2} \alpha_{1}+\alpha\left(\alpha_{1}+\alpha_{2}\right)+e_{1} n_{1}\left(M_{1}\left(H_{2}\right)+\bar{M}_{1}\left(H_{2}\right)\right)+4\left(e_{2}+\bar{e}_{2}\right)\left(2 \alpha_{3}+\alpha_{4}\right) .
\end{aligned}
$$

Proof. Using equation (4), we have

$$
\begin{aligned}
& \bar{F}\left(H_{1+S} H_{2}\right)=\sum_{\left(r_{1}, r_{2}\right)} \sum_{\left(s_{1}, s_{2}\right) \notin E\left(H_{1+S} H_{2}\right)}\left[d\left(r_{1}, s_{1}\right)^{2}+d\left(r_{2}, s_{2}\right)^{2}\right] \\
& \bar{F}\left(H_{1+S} H_{2}\right)=\left[\sum_{r_{1}, r_{2} \in\left(V\left(S H_{1}\right)-V\left(H_{1}\right)\right)}+\sum_{r_{1}, r_{2} \in V_{H_{1}}}+\sum_{\substack{r_{1}, r_{2} \in V\left(S\left(H_{1}\right)\right) \\
r_{1} \in V\left(H_{1}\right) \\
r_{2} \in V\left(S\left(H_{1}\right)-V\left(H_{1}\right)\right)}}\right] \sum_{s_{1}, s_{2} \in V_{H_{2}}}\left[d\left(r_{1}, s_{1}\right)^{2}+d\left(r_{2}, s_{2}\right)^{2}\right] \text {, } \\
& =\sum A+\sum B+\sum C \\
& \sum A=\sum_{r_{1}, r_{2} \in V\left(S\left(H_{1}\right)-V\left(H_{1}\right)\right)} \sum_{s_{1}, s_{2} \in V_{H_{2}}}\left[d_{S\left(H_{1}\right)}\left(r_{1}\right)+d_{S\left(H_{1}\right)}\left(r_{2}\right)\right]=\sum_{r_{1}, r_{2} \in V\left(S\left(H_{1}\right)-V\left(H_{1}\right)\right)} \sum_{s_{1}, s_{2} \in V_{H_{2}}}\left(2^{2}+2^{2}\right), \\
& \sum A=4\left(n_{2}^{2} e_{1}^{2}-n_{2} e_{1}\right) \\
& \sum B=\sum B_{1}+\sum B_{2}+\sum B_{3} \\
& \sum B_{1}=\sum_{r \in V_{H_{1}}} \sum_{s_{1} s_{2} \notin E_{H_{2}}}\left[d\left(r, s_{1}\right)^{2}+d\left(r, s_{2}\right)^{2}\right]=\sum_{r \in V_{H_{1}}} \sum_{s_{1} s_{2} \notin E_{H_{2}}}\left[\left(d(r)+d\left(s_{1}\right)\right)^{2}+\left(d(r)+d\left(s_{2}\right)\right)^{2}\right] \\
& =\sum_{r \in V_{H_{1}}} \sum_{s_{1} s_{2} \notin E_{H_{2}}}\left[\left(d(r)^{2}+d\left(s_{1}\right)^{2}+2 d(r) d\left(s_{1}\right)\right)+\left(d(r)^{2}+d\left(s_{2}\right)^{2}+2 d(r) d\left(s_{2}\right)\right)\right] \\
& =\sum_{r \in V_{H_{1}}} \sum_{s_{1} s_{2} \notin E_{H_{2}}}\left[2 d(r)^{2}+\left(d\left(s_{1}\right)^{2}+d\left(s_{2}\right)^{2}\right)+2 d(r)\left(d\left(s_{1}\right)+d\left(s_{2}\right)\right)\right] \\
& =2 \bar{e}_{2} M_{1}\left(H_{1}\right)+4 e_{1} \bar{M}_{1}\left(H_{2}\right)+n_{1} \bar{F}\left(H_{2}\right) \text {, } \\
& \sum B_{2} \sum_{s \in V_{H_{2}}} \sum_{r_{1}, r_{2} \in V_{H_{1}}}\left[\left(d\left(r_{1}\right)+d(s)\right)^{2}+\left(d\left(r_{2}\right)+d(s)\right)^{2}\right] \\
& =\sum_{s \in V_{H_{2}}}\left(\sum_{r_{1} r_{2} \in E_{H_{1}}}+\sum_{r_{1} r_{2} \notin E_{H_{1}}}\right)\left[\left(d\left(r_{1}\right)^{2}+d(s)^{2}+2 d\left(r_{1}\right) d(s)\right)+\left(d\left(r_{2}\right)^{2}+d(s)^{2}+2 d\left(r_{2}\right) d(s)\right)\right] \\
& \left.=\sum_{s \in V_{H_{2}}}\left(\sum_{r_{1} r_{2} \in E_{H_{1}}}+\sum_{r_{1} r_{2} \notin E_{H_{1}}}\right)\left[\left(d\left(r_{1}\right)^{2}+d\left(r_{2}\right)^{2}\right)+2 d(s)^{2}+2 d(s)\left(d\left(r_{1}\right)\right)+d\left(r_{2}\right)\right)\right]
\end{aligned}
$$




$$
\begin{aligned}
& =n_{2}\left(F\left(H_{1}\right)+\bar{F}\left(H_{1}\right)\right)+2 M_{1}\left(H_{2}\right)\left(e_{1}+\bar{e}_{1}\right)+4 e_{2}\left(M_{1}\left(H_{1}\right)+\bar{M}_{1}\left(H_{1}\right)\right) \text {, } \\
& \sum B_{3}=\left[\left(\sum_{r_{1} r_{2} \in E_{H_{1}}}+\sum_{r_{1} r_{2} \notin E_{H_{1}}}\right)\left(\sum_{s_{1} s_{2} \in E_{H_{2}}}+\sum_{s_{1} s_{2} \notin E_{H_{2}}}\right)\right]\left[d\left(r_{1}, s_{1}\right)^{2}+d\left(r_{2}, s_{2}\right)^{2}\right] \\
& =\left[\left(\sum_{r_{1} r_{2} \in E_{H_{1}}}+\sum_{r_{1} r_{2} \notin E_{H_{1}}}\right)\left(\sum_{s_{1} s_{2} \in E_{H_{2}}}+\sum_{s_{1} s_{2} \notin E_{H_{2}}}\right)\right]\left[\left(d\left(r_{1}\right)^{2}+d\left(r_{2}\right)^{2}\right)+2\left(d\left(r_{1}\right) d\left(s_{1}\right)+d\left(r_{2}\right) d\left(s_{2}\right)\right)+\left(d\left(s_{1}\right)^{2}+d\left(s_{2}\right)^{2}\right)\right] \\
& =2\left[\left(e_{2}+\bar{e}_{2}\right)\left(F\left(H_{1}\right)+\bar{F}\left(H_{1}\right)\right)+\left(e_{1}+\bar{e}_{1}\right)\left(F\left(H_{2}\right)+\bar{F}\left(H_{2}\right)\right)+\left(M_{1}\left(H_{2}\right)+\bar{M}_{1}\left(H_{2}\right)\right)\left(M_{1}\left(H_{1}\right)+\bar{M}_{1}\left(H_{1}\right)\right)\right] \text {, } \\
& \sum B=2 \bar{e}_{2} M_{1}\left(H_{1}\right)+4 e_{1} \bar{M}_{1}\left(H_{2}\right)+n_{1} \bar{F}\left(H_{2}\right)+n_{2}\left(F\left(H_{1}\right)+\bar{F}\left(H_{1}\right)+2 M_{1}\left(H_{2}\right)\left(e_{1}+\bar{e}_{1}\right)\right. \\
& +4 e_{2}\left(M_{1}\left(H_{1}\right)+\bar{M}_{1}\left(H_{1}\right)\right)+2\left[\left(e_{2}+\bar{e}_{2}\right)\left(F\left(H_{1}\right)+\bar{F}\left(H_{1}\right)\right)+\left(e_{1}+\bar{e}_{1}\right)\left(F\left(H_{2}\right)+\bar{F}\left(H_{2}\right)\right),\right. \\
& \sum C=\sum C_{1}+\sum C_{2}+\sum C_{3} \text {, } \\
& \sum C_{1}=\sum_{r_{1} r_{2} \notin E\left(s\left(H_{1}\right)\right)} \sum_{s \in V_{H_{2}}}\left[d\left(r_{1}, s\right)^{2}+d\left(r_{2}, s\right)^{2}\right] \\
& r_{1} \in V\left(H_{1}\right) \\
& r_{2} \in V\left(S\left(H_{1}\right)-V\left(H_{1}\right)\right) \\
& =\sum_{r_{1} r_{2} \notin E\left(S\left(H_{1}\right)\right)} \sum_{s \in V_{H_{2}}}\left[\left(d\left(r_{1}\right)^{2}+d(s)^{2}+2 d\left(r_{1}\right) d(s)\right)+\left(d_{S\left(H_{1}\right.}\left(r_{2}\right)^{2}\right)\right] \\
& r_{1} \in V\left(H_{1}\right) \\
& r_{2} \in V\left(S\left(H_{1}\right)-V\left(H_{1}\right)\right) \\
& =n_{2} \alpha_{1}+e_{1}\left(n_{1}-2\right) M_{1}\left(H_{2}\right)+4 e_{2} \alpha_{3}, \\
& \sum C_{2}=\sum_{r_{1} r_{2} \notin E\left(S\left(H_{1}\right)\right)} \sum_{s_{1}, s_{2} \in V_{H_{2}}}\left[d\left(r_{1}, s_{1}\right)^{2}+d\left(r_{2}, s_{2}\right)^{2}\right] \\
& r_{1} \in V\left(H_{1}\right) \\
& r_{2} \in V\left(S\left(H_{1}\right)-V\left(H_{1}\right)\right) \\
& =\sum_{r_{1} r_{2} \notin E\left(S\left(H_{1}\right)\right)}\left(\sum_{s_{1}, s_{2} \in V_{H_{2}}}+\sum_{s_{1}, s_{2} \notin V_{H_{2}}}\right)\left[\left(d\left(r_{1}\right)^{2}+d\left(s_{1}\right)^{2}+2 d\left(r_{1}\right) d\left(s_{1}\right)+\left(d_{S\left(H_{1}\right.}\left(r_{2}\right)^{2}\right]\right.\right. \\
& r_{1} \in V\left(H_{1}\right) \\
& r_{2} \in V\left(S\left(H_{1}\right)-V\left(H_{1}\right)\right) \\
& =\alpha \alpha_{1}+e_{1}\left(n_{1}-2\right)\left(M_{1}\left(H_{2}\right)+\bar{M}_{1}\left(H_{2}\right)\right)+4\left(e_{2}+\bar{e}_{2}\right) \alpha_{3}, \\
& \sum C_{3}=\sum_{r_{1} r_{2} \in E\left(S\left(H_{1}\right)\right)} \sum_{s_{1}, s_{2} \in V_{H_{2}}}\left[d\left(r_{1}, s_{1}\right)^{2}+d\left(r_{2}, s_{2}\right)^{2}\right] \\
& r_{1} \in V\left(H_{1}\right) \\
& r_{2} \in V\left(S\left(H_{1}\right)-V\left(H_{1}\right)\right) \\
& =\sum_{r_{1} r_{2} \in E\left(S\left(H_{1}\right)\right)}\left(\sum_{s_{1}, s_{2} \in V_{H_{2}}}+\sum_{s_{1}, s_{2} \notin V_{H_{2}}}\right)\left[\left(d\left(r_{1}\right)^{2}+d\left(s_{1}\right)^{2}+2 d\left(r_{1}\right) d\left(s_{1}\right)+\left(d_{S\left(H_{1}\right.}\left(r_{2}\right)^{2}\right]\right.\right. \\
& r_{1} \in V\left(H_{1}\right) \\
& r_{2} \in V\left(S\left(H_{1}\right)-V\left(H_{1}\right)\right) \\
& =\alpha \alpha_{2}+2 e_{1}\left(M_{1}\left(H_{2}\right)+\bar{M}_{1}\left(H_{2}\right)\right)+4\left(e_{2}+\bar{e}_{2}\right) \alpha_{4} .
\end{aligned}
$$


By substituting the values in equation (6), we get the required result.
Theorem 2. Let $H_{1+R} H_{2}$ be a $R$-sum graph; then, its $F$-coindex is

$$
\begin{aligned}
\bar{F}\left(H_{1+R} H_{2}\right)= & 4\left(n_{2}^{2} e_{1}^{2}-n_{2} e_{1}\right)+8 \bar{e}_{2} M_{1}\left(H_{1}\right)+8 e_{1} \bar{M}_{1}\left(H_{2}\right)+n_{1} \bar{F}\left(H_{2}\right) \\
& +4 n_{2}\left(\bar{F}\left(H_{1}\right)+\left(2 \bar{e}_{1}+\left(e_{1}\left(n_{1}-2\right)\right)\right)\right) M_{1}\left(H_{2}\right)+8 e_{2} \bar{M}_{1}\left(H_{1}\right) \\
& +24\left(e_{2}+\bar{e}_{2}\right)\left(F\left(H_{1}\right)+\bar{F}\left(H_{1}\right)\right)+\left(e_{1}+\bar{e}_{1}\right)\left(F\left(H_{2}\right)+\bar{F}\left(H_{2}\right)\right) \\
& \left.+2\left(M_{1}\left(H_{2}\right)+\bar{M}_{1}\left(H_{2}\right)\right)\left(M_{1}\left(H_{1}\right)+\bar{M}_{1}\left(H_{1}\right)\right)\right] \\
& +n_{2} \alpha_{1}+\alpha\left(\alpha_{1}+\alpha_{2}\right)+e_{1} n_{1}\left(M_{1}\left(H_{2}\right)+\bar{M}_{1}\left(H_{2}\right)\right)+4\left(e_{2}+\bar{e}_{2}\right)\left(2 \alpha_{3}+\alpha_{4}\right) .
\end{aligned}
$$

Proof. Using equation (4), we have

$$
\begin{aligned}
& \bar{F}\left(H_{1+R} H_{2}\right)=\sum_{\left(t_{1}, t_{2}\right)\left(x_{1}, x_{2}\right) \notin E\left(H_{1+S} H_{2}\right)}\left[d\left(t_{1}, x_{1}\right)^{2}+d\left(t_{2}, x_{2}\right)^{2}\right], \\
& \bar{F}\left(H_{1+R} H_{2}\right)=\left[\sum_{r_{1}, r_{2} \in\left(V\left(R\left(H_{1}\right)-V\left(H_{1}\right)\right)\right.}+\sum_{\substack{r_{1}, r_{2} \in V_{H_{1}} \\
r_{1}, r_{2} \in V\left(R\left(H_{1}\right)\right) \\
r_{1} \in V\left(H_{1}\right) \\
r_{2} \in V\left(R\left(H_{1}\right)-V\left(H_{1}\right)\right)}}+\sum_{s_{1}, s_{2} \in V_{H_{2}}}\left[d\left(r_{1}, s_{1}\right)^{2}+d\left(r_{2}, s_{2}\right)^{2}\right]\right. \\
& =\sum A+\sum B+\sum C \text {. }
\end{aligned}
$$

Using equation (7),

$$
\begin{aligned}
\sum A & =4\left(n_{2}^{2} e_{1}^{2}-n_{2} e_{1}\right), \\
\sum B & =\sum B_{1}+\sum B_{2}+\sum B_{3} \\
\sum B_{1} & =\sum_{r \in V_{H_{1}}} \sum_{s_{1} s_{2} \notin E_{H_{2}}}\left[d\left(r, s_{1}\right)^{2}+d\left(r, s_{2}\right)^{2}\right]=\sum_{r \in V_{H_{1}}} \sum_{s_{1} s_{2} \notin E_{H_{2}}}\left[\left(d(r)+d\left(s_{1}\right)\right)^{2}+\left(d(r)+d\left(s_{2}\right)\right)^{2}\right] \\
& \left.=\sum_{r \in V_{H_{1}}} \sum_{s_{1} s_{2} \notin E_{H_{2}}}\left[8 d(r)^{2}+\left(d\left(s_{1}\right)^{2}+d\left(s_{2}\right)^{2}\right)+4 d(r)\left(d\left(s_{1}\right)+d\left(s_{2}\right)\right)\right)\right] \\
& =8 \bar{e}_{2} M_{1}\left(H_{1}\right)+8 e_{1} \bar{M}_{1}\left(H_{2}\right)+n_{1} \bar{F}\left(H_{2}\right), \\
\sum B_{2} & =\sum_{s \in V_{H_{2}}} \sum_{r_{1}, r_{2} \in V_{H_{1}}}\left[\left(d\left(r_{1}\right)+d(s)\right)^{2}+\left(d\left(r_{2}\right)+d(s)\right)^{2}\right] \\
& =\sum_{s \in V_{H_{2}}}\left(\sum_{r_{1} r_{2} \in E_{H_{1}}}+\sum_{r_{1} r_{2} \notin E_{H_{1}}}\right)\left[4\left(d\left(r_{1}\right)^{2}+d\left(r_{2}\right)^{2}\right)+2 d(s)^{2}+4 d(s)\left(d\left(r_{1}\right)\right)+d\left(r_{2}\right)\right] \\
& =4 n_{2}\left(\bar{F}\left(H_{1}\right)+2 \bar{e}_{1} M_{1}\left(H_{2}\right)+8 e_{2} \bar{M}_{1}\left(H_{1}\right),\right. \\
\sum B_{3}= & {\left[\left(\sum_{r_{1} r_{2} \in E_{H_{1}}}+\sum_{r_{1} r_{2} \notin E_{H_{1}}}\right)\left(\sum_{s_{1} s_{2} \in E_{H_{2}}}+\sum_{s_{1} s_{2} \notin E_{H_{2}}}\right)\right]\left[d\left(r_{1}, s_{1}\right)^{2}+d\left(r_{2}, s_{2}\right)^{2}\right] }
\end{aligned}
$$




$$
\begin{aligned}
& =\left[\left(\sum_{r_{1} r_{2} \in E_{H_{1}}}+\sum_{r_{1} r_{2} \notin E_{H_{1}}}\right)\left(\sum_{s_{1} s_{2} \in E_{H_{2}}}+\sum_{s_{1} s_{2} \notin E_{H_{2}}}\right)\right]\left[\left(d\left(r_{1}\right)^{2}+d\left(r_{2}\right)^{2}\right)+\left(d\left(s_{1}\right)^{2}+d\left(s_{2}\right)^{2}+2\left(d\left(r_{1}\right) d\left(s_{1}\right)+d\left(r_{2}\right) d\left(s_{2}\right)\right)\right]\right. \\
& =\left[\left(\sum_{r_{1} r_{2} \in E_{H_{1}}}+\sum_{r_{1} r_{2} \notin E_{H_{1}}}\right)\left(\sum_{s_{1} s_{2} \in E_{H_{2}}}+\sum_{s_{1} s_{2} \notin E_{H_{2}}}\right)\right]\left[2^{2}\left(d\left(r_{1}\right)^{2}+d\left(r_{2}\right)^{2}\right)+\left(d\left(s_{1}\right)^{2}+d\left(s_{2}\right)^{2}+4\left(d\left(r_{1}\right) d\left(s_{1}\right)+d\left(r_{2}\right) d\left(s_{2}\right)\right)\right]\right. \\
& =2\left[4\left(e_{2}+\bar{e}_{2}\right)\left(F\left(H_{1}\right)+\bar{F}\left(H_{1}\right)\right)+\left(e_{1}+\bar{e}_{1}\right)\left(F\left(H_{2}\right)+\bar{F}\left(H_{2}\right)\right)+2\left(M_{1}\left(H_{2}\right)+\bar{M}_{1}\left(H_{2}\right)\right)\left(M_{1}\left(H_{1}\right)+\bar{M}_{1}\left(H_{1}\right)\right)\right] \\
& \sum B=8 \bar{e}_{2} M_{1}\left(H_{1}\right)+8 e_{1} \bar{M}_{1}\left(H_{2}\right)+n_{1} \bar{F}\left(H_{2}\right)+4 n_{2}\left(\bar{F}\left(H_{1}\right)+2 \bar{e}_{1} M_{1}\left(H_{2}\right)+8 e_{2} \bar{M}_{1}\left(H_{1}\right)\right. \\
& +2\left[4\left(e_{2}+\bar{e}_{2}\right)\left(F\left(H_{1}\right)+\bar{F}\left(H_{1}\right)\right)+\left(e_{1}+\bar{e}_{1}\right)\left(F\left(H_{2}\right)+\bar{F}\left(H_{2}\right)\right)\right. \\
& \left.+2\left(M_{1}\left(H_{2}\right)+\bar{M}_{1}\left(H_{2}\right)\right)\left(M_{1}\left(H_{1}\right)+\bar{M}_{1}\left(H_{1}\right)\right)\right] \text {, } \\
& \sum C=\sum C_{1}+\sum C_{2}+\sum C_{3} \\
& \sum C_{1}=\sum_{r_{1} r_{2} \notin E\left(R\left(H_{1}\right)\right)} \sum_{s \in V_{H_{2}}}\left[d\left(r_{1}, s\right)^{2}+d\left(r_{2}, s\right)^{2}\right] \\
& r_{1} \in V\left(H_{1}\right) \\
& r_{2} \in V\left(R\left(H_{1}\right)-V\left(H_{1}\right)\right) \\
& =\sum_{r_{1} r_{2} \notin E\left(R\left(H_{1}\right)\right)} \sum_{s \in V_{H_{2}}}\left[2^{2}\left(d\left(r_{1}\right)^{2}+d(s)^{2}+4 d\left(r_{1}\right) d(s)\right)+\left(d_{S\left(H_{1}\right.}\left(r_{2}\right)^{2}\right]\right. \\
& r_{1} \in V\left(H_{1}\right) \\
& r_{2} \in V\left(R\left(H_{1}\right)-V\left(H_{1}\right)\right) \\
& =n_{2} \alpha_{1}+e_{1}\left(n_{1}-2\right) M_{1}\left(H_{2}\right)+4 e_{2} \alpha_{3} \text {, } \\
& \sum C_{2}=\sum_{r_{1} r_{2} \notin E\left(R\left(H_{1}\right)\right)}\left(\sum_{s_{1}, s_{2} \in V_{H_{2}}}+\sum_{s_{1}, s_{2} \notin V_{H_{2}}}\right)\left[\left(d\left(r_{1}\right)^{2}+d\left(s_{1}\right)^{2}+2 d\left(r_{1}\right) d\left(s_{1}\right)+\left(d_{S\left(H_{1}\right.}\left(r_{2}\right)^{2}\right]\right.\right. \\
& r_{1} \in V\left(H_{1}\right) \\
& r_{2} \in V\left(R\left(H_{1}\right)-V\left(H_{1}\right)\right) \\
& =\alpha \alpha_{1}+e_{1}\left(n_{1}-2\right)\left(M_{1}\left(H_{2}\right)+\bar{M}_{1}\left(H_{2}\right)\right)+4\left(e_{2}+\bar{e}_{2}\right) \alpha_{3}, \\
& \sum C_{3}=\sum_{r_{1} r_{2} \in E\left(R\left(H_{1}\right)\right)}\left(\sum_{s_{1}, s_{2} \in V_{H_{2}}}+\sum_{s_{1}, s_{2} \notin V_{H_{2}}}\right)\left[\left(d\left(r_{1}\right)^{2}+d\left(s_{1}\right)^{2}+2 d\left(r_{1}\right) d\left(s_{1}\right)+\left(d_{S\left(H_{1}\right.}\left(r_{2}\right)^{2}\right]\right.\right. \\
& r_{1} \in V\left(H_{1}\right) \\
& r_{2} \in V\left(R\left(H_{1}\right)-V\left(H_{1}\right)\right) \\
& =\alpha \alpha_{2}+2 e_{1}\left(M_{1}\left(H_{2}\right)+\bar{M}_{1}\left(H_{2}\right)\right)+4\left(e_{2}+\bar{e}_{2}\right) \alpha_{4} .
\end{aligned}
$$

By substituting the values in equation (10), we get the required result. 
Theorem 3. Let $H_{1+Q} H_{2}$ be a Q-sum graph; then, F-coindex is

$$
\begin{aligned}
\bar{F}\left(H_{1+Q} H_{2}\right)= & n_{2} \bar{F}\left(Q^{\prime}\left(G_{1}\right)\right)+2\left(\bar{e}_{2}+\left(n_{2}-1\right)\right)\left(M_{1}\left(Q^{\prime} H_{1}\right)+1\right) F\left(Q^{\prime}\left(G_{1}\right)\right. \\
& \left.+\bar{F}\left(Q^{\prime}\left(G_{1}\right)\right)\right)+2 \bar{e}_{2} M_{1}\left(H_{1}\right)+4 e_{1} \bar{M}_{1}\left(H_{2}\right)+n_{1} \bar{F}\left(H_{2}\right)+n_{2}\left(F\left(H_{1}\right)+\bar{F}\left(H_{1}\right)\right) \\
& +2 M_{1}\left(H_{2}\right)\left(e_{1}+\bar{e}_{1}\right)+4 e_{2}\left(M_{1}\left(H_{1}\right)+\bar{M}_{1}\left(H_{1}\right)\right)+2\left[\left(e_{2}+\bar{e}_{2}\right)\left(F\left(H_{1}\right)+\bar{F}\left(H_{1}\right)\right)\right. \\
& +\left(e_{1}+\bar{e}_{1}\right)\left(F\left(H_{2}\right)+\bar{F}\left(H_{2}\right)\right)+n_{2} \bar{F}\left(Q\left(H_{1}^{\prime}\right)\right)+e_{1}\left(n_{1}-2\right) M_{1}\left(H_{2}\right) \\
& +\alpha\left(\alpha_{1}+\alpha_{2}\right)+e_{1} n_{1}\left(M_{1}\left(H_{2}\right)+\bar{M}_{1}\left(H_{2}\right)\right)+4\left(e_{2}+\bar{e}_{2}\right)\left(2 \alpha_{3}+\alpha_{4}\right) .
\end{aligned}
$$

Proof. Using equation (4), we have

$$
\begin{aligned}
& \bar{F}\left(H_{1+Q} H_{2}\right)=\sum_{\left(t_{1}, t_{2}\right)}\left[d\left(t_{1}, x_{2}\right) \notin E\left(x_{1+Q} H_{2}\right)+d\left(t_{2}, x_{2}\right)^{2}\right],
\end{aligned}
$$

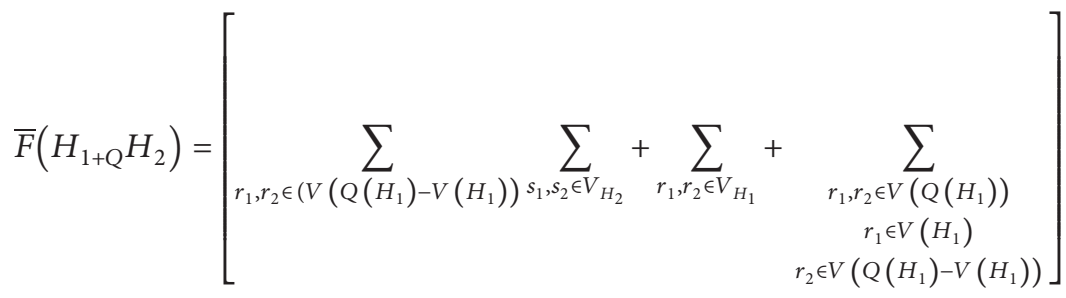

$$
\begin{aligned}
& \text {. } \sum_{s_{1}, s_{2} \in V_{H_{2}}}\left[d\left(r_{1}, s_{1}\right)^{2}+d\left(r_{2}, s_{2}\right)^{2}\right] \\
& =\sum A+\sum B+\sum C, \\
& \sum A=\sum A_{1}+\sum A_{2}+\sum A_{3}+\sum A_{4}, \\
& \sum A_{1}=\sum_{\substack{r_{1} r_{2} \notin E\left(Q\left(H_{1}\right)\right) \\
r_{1}, r_{2} \in V\left(Q\left(H_{1}\right)-H_{1}\right)}} \sum_{s \in V_{H_{2}}}\left[d_{Q\left(H_{1}\right.}\left(r_{1}\right)^{2}+d_{Q\left(H_{1}\right.}\left(r_{2}\right)^{2}\right]=n_{2} \text {, } \\
& \sum_{r_{1} r_{2} \notin E\left(Q\left(H_{1}\right)\right)}\left[d_{Q\left(H_{1}\right.}\left(r_{1}\right)^{2}+d_{Q\left(H_{1}\right.}\left(r_{2}\right)^{2}\right] \\
& r_{1}, r_{2} \in V\left(Q\left(H_{1}\right)-H_{1}\right)
\end{aligned}
$$$$
\text { if } \sum_{\substack{r_{1} r_{2} \notin E\left(Q\left(H_{1}\right)\right) \\ r_{1}, r_{2} \in V\left(Q\left(H_{1}\right)-H_{1}\right)}}\left[d_{Q\left(H_{1}\right.}\left(r_{1}\right)^{2}+d_{Q\left(H_{1}\right.}\left(r_{2}\right)^{2}\right]=\bar{F}\left(Q^{\prime}\left(H_{1}\right)\right) \text {, then } \sum A_{1}=n_{2} \bar{F}\left(Q^{\prime}\left(H_{1}\right)\right) \text {, }
$$

$r_{1}, r_{2} \in V\left(Q\left(H_{1}\right)-H_{1}\right)$

$$
\begin{aligned}
\sum A_{2} & =\sum_{r \in V\left(Q\left(H_{1}\right)-\left(H_{1}\right)\right)} \sum_{s_{1} s_{2} \in E_{H_{2}}}\left[d_{Q\left(H_{1}\right.}\left(r_{1}\right)^{2}+d_{Q\left(H_{1}\right.}\left(r_{2}\right)^{2}\right] \\
& =\sum_{r \in V\left(Q\left(H_{1}\right)-V\left(H_{1}\right)\right)}\left(\sum_{s_{1} s_{2} \notin E_{H_{2}}}+\sum_{s_{1} s_{2} \in E_{H_{2}}}\right)\left[d_{Q\left(H_{1}\right.}\left(r_{1}\right)^{2}+d_{Q\left(H_{1}\right.}\left(r_{2}\right)^{2}\right] \\
& \left.=2\left(\bar{e}_{2}+\left(n_{2}-1\right)\right) \sum_{r \in V\left(Q\left(H_{1}\right)-V\left(H_{1}\right)\right)}\left[d_{Q\left(H_{1}\right.}(r)^{2}\right)\right]
\end{aligned}
$$




$$
\text { if } \begin{aligned}
\left.\sum_{r \in V\left(Q\left(H_{1}\right)-\left(H_{1}\right)\right)}\left[d_{Q\left(H_{1}\right.}(r)^{2}\right)\right]= & M_{1}\left(Q \prime H_{1}\right) \text {, then }=2\left(\bar{e}_{2}+\left(n_{2}-1\right)\right) M_{1}\left(Q^{\prime} H_{1}\right), \\
\sum A_{3}= & \sum_{\substack{r_{1} r_{2} \notin E\left(Q\left(H_{1}\right)\right) \\
r_{1}, r_{2} \in V\left(Q\left(H_{1}\right)-H_{1}\right)}} \sum_{s_{1}, s_{2} \in V_{H_{2}}}\left[d_{Q\left(H_{1}\right.}\left(r_{1}\right)^{2}+d_{Q\left(H_{1}\right.}\left(r_{2}\right)^{2}\right] \\
= & \sum_{\substack{r_{1} r_{2} \notin E\left(Q\left(H_{1}\right)\right) \\
r_{1}, r_{2} \in V\left(Q\left(H_{1}\right)-V\left(H_{1}\right)\right)}}\left[\sum_{s_{1} s_{2} \in E_{H_{2}}}\left(d_{Q\left(H_{1}\right.}\left(r_{1}\right)^{2}+d_{Q\left(H_{1}\right.}\left(r_{2}\right)^{2}\right)\right. \\
& +\sum_{\substack{s_{1} s_{2} \notin E_{H_{2}} \\
=}}\left(d_{Q\left(\bar{e}_{2}+\left(n_{1}-1\right)\right)} \sum_{\substack{r_{1} r_{2} \notin E\left(Q\left(H_{1}\right)\right) \\
r_{1}, r_{2} \in V\left(Q\left(H_{1}\right)-V\left(H_{1}\right)\right)}}\left[d_{Q\left(H_{1}\right.}\left(r_{1}\right)^{2}+d_{Q\left(H_{1}\right.}\left(r_{2}\right)^{2}\right]\right.
\end{aligned}
$$

if $\sum_{r_{1} r_{2} \notin E\left(Q\left(H_{1}\right)\right)} \quad\left[d_{Q\left(H_{1}\right.}\left(r_{1}\right)^{2}+d_{Q\left(H_{1}\right.}\left(r_{2}\right)^{2}\right]=F\left(Q^{\prime}\left(H_{1}\right)\right)=2\left(\bar{e}_{2}+\left(n_{2}-1\right)\right) F\left(Q^{\prime}\left(H_{1}\right)\right)$,

$r_{1}, r_{2} \in V\left(Q\left(H_{1}\right)-V\left(H_{1}\right)\right)$

$$
\begin{aligned}
\sum A_{4}= & \sum_{\substack{r_{1} r_{2} \notin E\left(Q\left(H_{1}\right)\right) \\
r_{1}, r_{2} \in V\left(Q\left(H_{1}\right)-V\left(H_{1}\right)\right)}} \sum_{s_{1}, s_{2} \in V_{H_{2}}}\left[d_{Q\left(H_{1}\right.}\left(r_{1}\right)^{2}+d_{Q\left(H_{1}\right.}\left(r_{2}\right)^{2}\right] \\
= & \sum_{\substack{r_{1} r_{2} \notin E\left(Q\left(H_{1}\right)\right) \\
r_{1}, r_{2} \in V\left(Q\left(H_{1}\right)-V\left(H_{1}\right)\right)}}\left[\sum_{s_{1} s_{2} \in E_{H_{2}}}\left(d_{Q\left(H_{1}\right.}\left(r_{1}\right)^{2}+d_{Q\left(H_{1}\right.}\left(r_{2}\right)^{2}\right)\right. \\
& \left.+\sum_{s_{1} s_{2} \notin E_{H_{2}}}\left(d_{Q\left(H_{1}\right.}\left(r_{1}\right)^{2}+d_{Q\left(H_{1}\right.}\left(r_{2}\right)^{2}\right)\right] \\
= & 2\left(\bar{e}_{2}+\left(n_{2}-1\right)\right) \sum_{\substack{r_{1} r_{2} \notin E\left(Q\left(H_{1}\right)\right) \\
r_{1}, r_{2} \in V\left(Q\left(H_{1}\right)-V\left(H_{1}\right)\right)}}\left[d_{Q\left(H_{1}\right.}\left(r_{1}\right)^{2}+d_{Q\left(H_{1}\right.}\left(r_{2}\right)^{2}\right] .
\end{aligned}
$$

Take $\sum_{\substack{r_{1} r_{2} \notin E\left(Q\left(H_{1}\right)\right) \\ r_{1}, r_{2} \in V\left(Q\left(H_{1}\right)-V\left(H_{1}\right)\right)}}\left[d_{Q\left(H_{1}\right.}\left(r_{1}\right)^{2}+d_{Q\left(H_{1}\right.}\left(r_{2}\right)^{2}\right]=\bar{F}\left(Q^{\prime}\left(H_{1}\right)\right)$, then $=2\left(\bar{e}_{2}+\left(n_{2}-1\right)\right) \bar{F}\left(Q^{\prime}\left(H_{1}\right)\right)$.

Consequently,

$$
\sum A=n_{2} \bar{F}\left(Q^{\prime}\left(G_{1}\right)\right)+2\left(\bar{e}_{2}+\left(n_{2}-1\right)\right) M_{1}\left(Q^{\prime} H_{1}\right)+2\left(\bar{e}_{2}+\left(n_{2}-1\right)\right) F\left(Q^{\prime}\left(G_{1}\right)+\bar{F}\left(Q^{\prime}\left(G_{1}\right)\right)\right) .
$$


TABLE 1: For two graphs, $H_{1} \cong P_{n}$ and $H_{2} \cong P_{m}$ values of forgotten coindex for their $D$-sum graphs by taking different values for $n$ and $m$.

\begin{tabular}{lcccc}
\hline$n, m$ & $\bar{F}\left(P_{n+S} P_{m}\right)$ & $\bar{F}\left(P_{n+R} P_{m}\right)$ & $\bar{F}\left(P_{n+Q} P_{m}\right)$ & $\bar{F}\left(P_{n+T} P_{m}\right)$ \\
\hline 3,2 & 332 & 528 & 410 & 632 \\
4,2 & 840 & 1268 & 1760 & 1820 \\
5,2 & 1476 & 2552 & 3214 & 4002 \\
6,2 & 2276 & 4585 & 4160 & 6552 \\
7,2 & 3332 & 6448 & 7034 & 9830 \\
8,2 & 4840 & 9512 & 9514 & 14362 \\
\hline
\end{tabular}

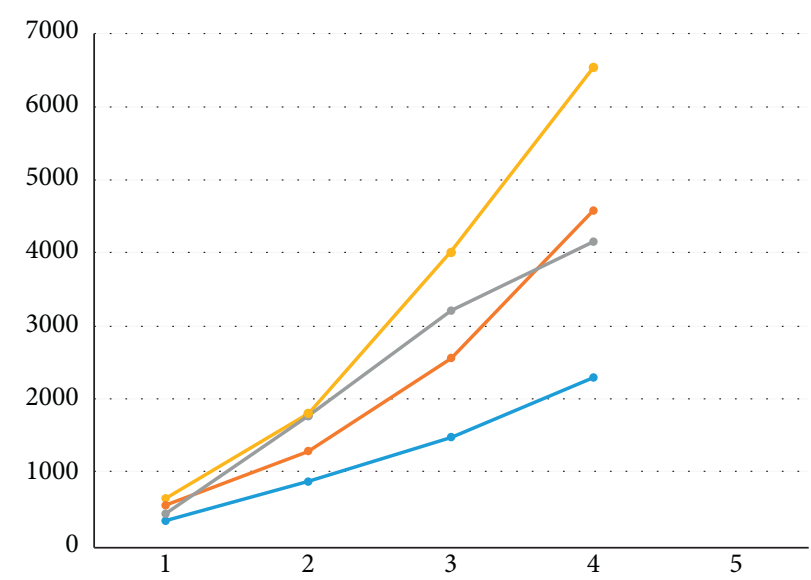

Figure 3: Graphical presentations of $F$-coincides for $P_{n+D} P_{m}$ graphs such as $H_{1+S} H_{2}, H_{1+R} H_{2}, H_{1+Q} H_{2}$, and $H_{1+T} H_{2}$ are represented by blue, orange, gray, and yellow, respectively.

Using equation (8), we directly have

$$
\begin{aligned}
& \sum B=2 \bar{e}_{2} M_{1}\left(H_{1}\right)+4 e_{1} \bar{M}_{1}\left(H_{2}\right)+n_{1} \bar{F}\left(H_{2}\right)+n_{2}\left(F\left(H_{1}\right)+\bar{F}\left(H_{1}\right)+2 M_{1}\left(H_{2}\right)\left(e_{1}+\bar{e}_{1}\right)\right. \\
& +4 e_{2}\left(M_{1}\left(H_{1}\right)+\bar{M}_{1}\left(H_{1}\right)\right)+2\left[\left(e_{2}+\bar{e}_{2}\right)\left(F\left(H_{1}\right)+\bar{F}\left(H_{1}\right)\right)+\left(e_{1}+\bar{e}_{1}\right)\left(F\left(H_{2}\right)+\bar{F}\left(H_{2}\right)\right),\right. \\
& \sum C=\sum C_{1}+\sum C_{2}+\sum C_{3} \text {, } \\
& \sum C_{1}=\sum_{r_{1} r_{2} \notin E\left(s\left(H_{1}\right)\right)} \sum_{s \in V_{H_{2}}}\left[d\left(r_{1}, s\right)^{2}+d\left(r_{2}, s\right)^{2}\right] \\
& r_{1} \in V\left(H_{1}\right) r_{2} \in V\left(Q\left(H_{1}\right)-V\left(H_{1}\right)\right)
\end{aligned}
$$

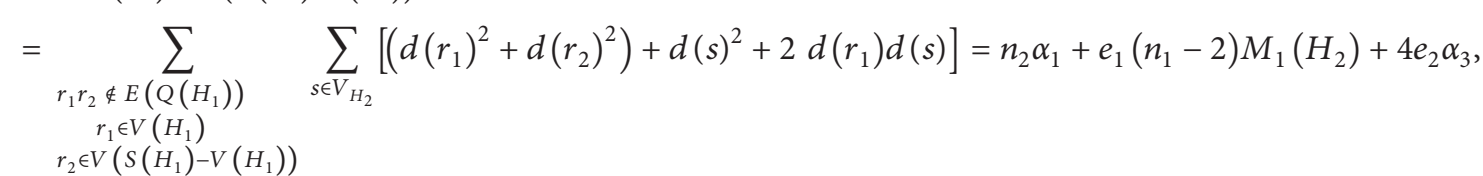

$$
\begin{aligned}
& \sum C_{2}=\sum_{\substack{r_{1} r_{2} \notin E\left(Q\left(H_{1}\right)\right) \\
r_{1} \in V\left(H_{1}\right) \\
r_{2} \in V\left(S\left(H_{1}\right)-V\left(H_{1}\right)\right)}}\left(\sum_{s_{1}, s_{2} \in V_{H_{2}}}+\sum_{s_{1} s_{2} \notin E_{H_{2}}}\right)\left[\left(d\left(r_{1}, s_{1}\right)^{2}+d\left(r_{2}, s_{2}\right)^{2}\right)\right] \\
& =\alpha \alpha_{1}+e_{1}\left(n_{1}-2\right)\left(M_{1}\left(H_{2}\right)+\bar{M}_{1}\left(H_{2}\right)\right)+4\left(e_{2}+\bar{e}_{2}\right) \alpha_{3}, \\
& \sum C_{3}=\sum_{r_{1} r_{2} \in E\left(Q\left(H_{1}\right)\right)}\left(\sum_{s_{1}, s_{2} \in V_{H_{2}}}+\sum_{s_{1}, s_{2} \notin V_{H_{2}}}\right)\left[\left(d\left(r_{1}, s_{1}\right)^{2}+d\left(r_{2}, s_{2}\right)^{2}\right)\right] \\
& r_{1} \in V\left(H_{1}\right) r_{2} \in V\left(S\left(H_{1}\right)-V\left(H_{1}\right)\right) \\
& =\alpha \alpha_{2}+2 e_{1}\left(M_{1}\left(H_{2}\right)+\bar{M}_{1}\left(H_{2}\right)\right)+4\left(e_{2}+\bar{e}_{2}\right) \alpha_{4} \text {. }
\end{aligned}
$$


By substituting the values in equation (13), we get the required result.
Theorem 4. Let $H_{1+T} H_{2}$ be a T-sum graph; then, its F-coindex is

$$
\begin{aligned}
\bar{F}\left(H_{1+T} H_{2}\right)= & n_{2} \bar{F}\left(Q^{\prime}\left(G_{1}\right)\right)+2\left(\bar{e}_{2}+\left(n_{2}-1\right)\right)\left(M_{1}\left(Q^{\prime} H_{1}\right)+1\right) F\left(Q^{\prime}\left(G_{1}\right)\right) \\
& +2\left(\bar{e}_{2}+\left(n_{2}-1\right)\right) \bar{F}\left(Q^{\prime}\left(G_{1}\right)\right)+\bar{e}_{2} M_{1}\left(H_{1}\right)+8 e_{1} \bar{M}_{1}\left(H_{2}\right)+n_{1} \bar{F}\left(H_{2}\right) \\
& +4 n_{2}\left(\bar{F}\left(H_{1}\right)+2 \bar{e}_{1} M_{1}\left(H_{2}\right)+8 e_{2} \bar{M}_{1}\left(H_{1}\right)+2\left[4 ( e _ { 2 } + \overline { e } _ { 2 } ) \left(F\left(H_{1}\right)\right.\right.\right. \\
& \left.+\bar{F}\left(H_{1}\right)\right)+\left(e_{1}+\bar{e}_{1}\right)\left(F\left(H_{2}\right)+\bar{F}\left(H_{2}\right)\right)+2\left(M_{1}\left(H_{2}\right)+\bar{M}_{1}\left(H_{2}\right)\right)\left(M_{1}\left(H_{1}\right)\right. \\
& \left.\left.d+\bar{M}_{1}\left(H_{1}\right)\right)\right]+n_{2} \bar{F}\left(T\left(H_{1}^{\prime}\right)\right)+e_{1}\left(n_{1}-2\right) M_{1}\left(H_{2}\right)+\alpha\left(\alpha_{1}+\alpha_{2}\right) \\
& +e_{1} n_{1}\left(M_{1}\left(H_{2}\right)+\bar{M}_{1}\left(H_{2}\right)\right)+4\left(e_{2}+\bar{e}_{2}\right)\left(2 \alpha_{3}+\alpha_{4}\right) .
\end{aligned}
$$

Proof. It follows from Theorems 2 and 3.

\section{Conclusion}

In this study, we have computed forgotten coindex of $D$-sum graphs such as $\bar{F}\left(H_{1+S} H_{2}\right), \bar{F}\left(H_{1+R} H_{2}\right), \bar{F}\left(H_{1+Q} H_{2}\right)$, and $\bar{F}\left(H_{1+T} H_{2}\right)$ in their general forms. If $H_{1}=P_{n}$ and $H_{2}=P_{m}$ of order $n, m \geq 2$, then

(i) In this study, we computed the exact values of forgotten coindex of $D$-sum graphs such as $\left(H_{1+D} H_{2}\right)$, where $D \in\{S, R, Q, T\}$ in the form of forgotten index, first Zagreb indices, and coincides their basic graphs $H_{1}$ and $H_{2}$.

(ii) We illustrated Theorems 1-4 with the help of some particular path graphs such as $P_{n+S} P_{m}, P_{n+R} P_{m}$, $P_{n+Q} P_{m}$, and $P_{n+T} P_{m}$ for $n, m \geq 2$. Forgotten coindex of $D$-sum graphs from the paths is also computed as applications of the obtained results.

(iii) Table 1 and Figure 3 present that forgotten coindex of $\left(P_{n+T} P_{m}\right)$ is dominant than forgotten coindex of $P_{n+S} P_{m}, P_{n+R} P_{m}$, and $P_{n+Q} P_{m}$.

$$
\begin{aligned}
& \bar{F}\left(P_{m+S} P_{n}\right)=4 m(n-1)(m(n-1)-1)+(m-1)(m-2) M\left(P_{n}\right)+4(n-1) \bar{M} P_{m}+n \bar{F}\left(P_{m}\right) \\
& +m\left(F \left(P_{n}+\bar{F}\left(P_{n}\right)+n(n-1) M_{1}\left(P_{m}\right)+4(m-1)\left(M_{1}(P n)+\bar{M}_{1}(P n)\right)\right.\right. \\
& +m(m-1)\left(F\left(P_{n}\right)+\bar{F}\left(P_{n}\right)\right)+n(n-1)\left(F\left(P_{m}\right)+\bar{F}\left(P_{m}\right)\right)+2\left(M_{1}\left(P_{m}\right)+\bar{M}_{1}\left(P_{m}\right)\right)\left(M_{1}\left(P_{n}\right)\right. \\
& \left.+\bar{M}_{1}\left(P_{n}\right)\right)+m \bar{F}\left(S\left(P_{n}^{\prime}\right)\right)+(n-1)(n-2) M_{1}\left(P_{m}\right)+4(m-1) \bar{B}\left(S\left(P_{n}^{\prime}\right)\right)+\alpha \bar{F}\left(S\left(P_{\prime}^{\prime}\right)_{n}\right) \\
& +(n-1)(n-2)\left(M_{1}\left(P_{m}\right)+\bar{M}_{1}\left(P_{m}\right)\right)+2 m(m-1) \bar{\beta}\left(S\left(P_{n}^{\prime}\right)\right)+\alpha F\left(S\left(P_{n}^{\prime}\right)\right) \\
& +2(n-1)\left(M_{1}\left(P_{m}\right)+\bar{M}_{1}\left(P_{m}\right)\right)+2 m(m-1) \beta\left(S\left(P_{n}^{\prime}\right)\right), \\
& \bar{F}\left(P_{m+R} P_{n}\right)=4 m(n-1)(m(n-1)-1)+4(m-1)(m-2) M\left(P_{n}\right)+8(n-1) \bar{M} P_{m}+n \bar{F}\left(P_{m}\right) \\
& +4 m \bar{F}\left(P_{n}+n(n-1) M_{1}\left(P_{m}\right)+8(m-1) \bar{M}_{1}(P n)+4 m(m-1)\left(F\left(P_{n}\right)+\bar{F}\left(P_{n}\right)\right)+n(n-1)\left(F\left(P_{m}\right)\right.\right. \\
& \left.+\bar{F}\left(P_{m}\right)\right)+4\left(M_{1}\left(P_{m}\right)+\bar{M}_{1}\left(P_{m}\right)\right)\left(M_{1}\left(P_{n}\right)+\bar{M}_{1}\left(P_{n}\right)\right)+m \bar{F}\left(R\left(P_{n}^{\prime}\right)\right)+(n-1)(n-2) M_{1}\left(P_{m}\right) \\
& +4(m-1) \bar{\beta}\left(R\left(P_{n}^{\prime}\right)\right)+\alpha \bar{F}\left(R\left(P_{\prime}\right)_{n}\right)+(n-1)(n-2)\left(M_{1}\left(P_{m}\right)+\bar{M}_{1}\left(P_{m}\right)\right)+2 m(m-1) \bar{\beta}\left(R\left(P_{n}^{\prime}\right)\right) \\
& +\alpha F\left(R\left(P_{n}^{\prime}\right)\right)+2(n-1)\left(M_{1}\left(P_{m}\right)+\bar{M}_{1}\left(P_{m}\right)\right)+2 m(m-1) \beta\left(R\left(P_{n}^{\prime}\right)\right), \\
& \bar{F}\left(P_{m+Q} P_{n}\right)=n \bar{F}\left(Q^{\prime} P_{n}\right)+2(m+n-2)\left(M_{1}\left(Q_{\prime} H_{1}\right)+\bar{F}\left(Q_{\prime} P_{n}\right)+F\left(Q \prime P_{n}\right)\right) \\
& +(m-1)(m-2) M_{P_{n}}+4(n-1) \bar{M}_{P_{m}}+n \bar{F}\left(P_{m}\right)+m\left(F \left(P_{n}+\bar{F}\left(P_{n}\right)+n(n-1) M_{1}\left(P_{m}\right)\right.\right. \\
& +4(m-1)\left(M_{1}(P n)+\bar{M}_{1}(P n)\right)+m(m-1)\left(F\left(P_{n}\right)+\bar{F}\left(P_{n}\right)\right)+n(n-1)\left(F\left(P_{m}\right)+\bar{F}\left(P_{m}\right)\right)
\end{aligned}
$$




$$
\begin{aligned}
+ & 2\left(M_{1}\left(P_{m}\right)+\bar{M}_{1}\left(P_{m}\right)\right)\left(M_{1}\left(P_{n}\right)+\bar{M}_{1}\left(P_{n}\right)\right)+m \bar{F}\left(Q\left(P_{n}^{\prime}\right)\right)+(n-1)(n-2) M_{1}\left(P_{m}\right) \\
+ & 4(m-1) \bar{\beta}\left(S\left(P_{n}^{\prime}\right)\right)+\alpha \bar{F}\left(S\left(Q^{\prime}\right)_{n}\right)+2 m(m-1) \bar{\beta}\left(S\left(Q_{n}^{\prime}\right)\right) \\
+ & (n-1)(n-2)\left(M_{1}\left(P_{m}\right)+\bar{M}_{1}\left(P_{m}\right)\right)+\alpha F\left(Q\left(P_{n}^{\prime}\right)\right)+2(n-1)\left(M_{1}\left(P_{m}\right)+\bar{M}_{1}\left(P_{m}\right)\right) \\
+ & 2 m(m-1) \beta\left(Q\left(P_{n}^{\prime}\right)\right), \\
\bar{F}\left(P_{m+T} P_{n}\right)= & n \bar{F}\left(Q^{\prime} P_{n}\right)+2(m+n-2)\left(M_{1}\left(Q^{\prime} H_{1}\right)+\bar{F}\left(Q_{\prime}^{\prime} P_{n}\right)+F\left(Q^{\prime} P_{n}\right)\right) \\
+ & 4(m-1)(m-2) M_{P_{n}}+8(n-1) \bar{M}_{P_{m}}+n \bar{F}\left(P_{m}\right)+4 m \bar{F}\left(P_{n}+n(n-1) M_{1}\left(P_{m}\right)+8(m-1) \bar{M}_{1}\left(P_{n}\right)\right. \\
+ & 4 m(m-1)\left(F\left(P_{n}\right)+\bar{F}\left(P_{n}\right)\right)+n(n-1)\left(F\left(P_{m}\right)+\bar{F}\left(P_{m}\right)\right)+4\left(M_{1}\left(P_{m}\right)+\bar{M}_{1}\left(P_{m}\right)\right)\left(M_{1}\left(P_{n}\right)\right. \\
+ & \left.\bar{M}_{1}\left(P_{n}\right)\right)+m \bar{F}\left(T\left(P_{n}^{\prime}\right)\right)+(n-1)(n-2) M_{1}\left(P_{m}\right)+4(m-1) \bar{\beta}\left(\beta\left(P_{n}^{\prime}\right)\right)+\alpha \bar{F}\left(T\left(P^{\prime}\right)_{n}\right) \\
+ & 2 m(m-1) \beta\left(S\left(P_{n}^{\prime}\right)\right)+(n-1)(n-2)\left(M_{1}\left(P_{m}\right)+\bar{M}_{1}\left(P_{m}\right)\right)+2 m(m-1) \bar{\beta}\left(T\left(P_{n}^{\prime}\right)\right) \\
+ & \alpha F\left(T\left(P_{n}^{\prime}\right)\right)+2(n-1)\left(M_{1}\left(P_{m}\right)+\bar{M}_{1}\left(P_{m}\right)\right) .
\end{aligned}
$$

Open problem 1. Investigate the existence of the distancebased coindices for the $D$-sum graphs.

\section{Data Availability}

The data used to support the findings of this study are included within the article and are available from the corresponding author upon request.

\section{Conflicts of Interest}

The authors declare that they have no conflicts of interest.

\section{Acknowledgments}

The authors are very thankful for the referees' comments, which helped them to improve the results and presentation of the manuscript to a great extent. This study was partially supported by University of Tabuk, Tabuk, Saudi Arabia.

\section{References}

[1] A. R. Matamala and E. Estrada, "Generalised topological indices: optimisation methodology and physico-chemical interpretation," Chemical Physics Letters, vol. 410, pp. 343$347,2005$.

[2] G. Rücker and C. Rücker, "On topological indices, boiling points, and cycloalkanes," Journal of Information and Computer Sciences, vol. 39, pp. 788-802, 1999.

[3] I. Gutman, "Degree-based topological indices," Croatica Chemica Acta, vol. 4, pp. 351-361, 2013.

[4] H. Wiener, "Structural determination of paraffin boiling points," Journal of the American Chemical Society, vol. 69, no. 1, pp. 17-20, 1947.

[5] I. Gutman and N. Trinajstic, "Graph theory and molecular orbitals. Total energy of alternant hydrocarbons," Chemical Physics Letters, vol. 4, pp. 535-538, 1972.

[6] D. H. Rouvray, "The modeling of chemical phenomena using topological indices," Journal of Computational Chemistry, vol. 8, no. 4, pp. 470-480, 1987.
[7] A. T. Balaban, "Topological indices based on topological distances in molecular graphs," Pure and Applied Chemistry, vol. 55, no. 2, pp. 199-206, 1983.

[8] A. T. Balaban, I. Motoc, D. Bonchev, and O. Mekenyan, "Topological indices for structureactivity correlations," Steric effects in drug design, pp. 21-55, Springer, Berlin, Germany, 1983.

[9] E. Cornwell, "New idea for the topological index evaluation and treatise multiple regression with three independent variables. Saturated Hydrocarbons used like a model," Journal of the Chilean Chemical Society, vol. 1, no. 1, pp. 765-768, 2006.

[10] D. J. Klein, Z. Mihalic, D. Plavsic, and N. Trinajstic, "Molecular topological index: a relation with the Wiener index," Journal of Chemical Information and Computer Sciences, vol. 32, no. 4, pp. 304-305, 1992.

[11] S. Mendiratta and A. K. Madan, "Structure-activity study on antiviral 5-vinylpyrimidine nucleoside analogs using Wiener's topological index," Journal of Chemical Information and Computer Sciences, vol. 34, no. 4, pp. 867-871, 1994.

[12] I. Gutman and E. Estrada, "Topological indices based on the line graph of the molecular graph," Journal of Chemical Information and Computer Sciences, vol. 36, no. 3, pp. 541-543, 1996.

[13] R. Biye, X. You, and C. Guobin, “A novel topological index for QSPR/QSAR study of organic compounds," Acta Chimica Sinica, vol. 57, no. 6, pp. 563-571, 1999.

[14] W. Qinghua, F. Xinlu, Y. Guanhan, Y. Xiaodong, and T. Chengling, "Study on a prediction method for Octane number of acyclic alkane by autocorrelation topological index," Journal of Petrochemical Universities, vol. 13, no. 3, pp. 50-53, 2000.

[15] A. Q. Baig, M. Imran, and H. Ali, "On topological indices of poly oxide, poly silicate, DOX, and DSL networks," Canadian Journal of Chemistry, vol. 93, no. 7, pp. 730-739, 2015.

[16] B. Furtula and I. Gutman, "A forgotten topological index," Journal of Mathematical Chemistry, vol. 53, no. 4, pp. 11841190, 2015.

[17] I. Gutman, A. Ghalavand, T. Dehghan-Zadeh, and A. R. Ashrafi, "Graphs with smallest forgotten index," Iranian 
Journal of Mathematical Chemistry, vol. 8, no. 3, pp. 259-273, 2017.

[18] Z. Che and Z. Chen, "Lower and upper bounds of the forgotten topological index," MATCH Communications in Mathematical and in Computer Chemistry, vol. 76, no. 3, pp. 635-648, 2016.

[19] W. Gao, M. K. Siddiqui, M. Imran, M. K. Jamil, and M. R. Farahani, "Forgotten topological index of chemical structure in drugs," Saudi Pharmaceutical Journal, vol. 24, no. 3, pp. 258-264, 2016.

[20] J. B. Liu, M. M. Matejic, E. I. Milovanovic, and I. Z. Milovanovic, "Some new inequalities for the forgotten topological index and coindex of graphs," MATCH Communications in Mathematical and in Computer Chemistry, vol. 84, no. 3, pp. 719-738, 2020.

[21] A. Ahmad, K. Elahi, R. Hasni, and M. F. Nadeem, "Computing the degree based topological indices of line graph of benzene ring embedded in P-type-surface in 2D network," Journal of Information and Optimization Sciences, vol. 40, no. 7, pp. 1511-1528, 2019.

[22] M. Javaid, M. Ahmad, M. Hussain, and W. C. Teh, "Bounds of F-index for unicyclic graphs with fixed pendent vertices," Journal of Prime Research in Mathematics, vol. 14, pp. 51-61, 2018.

[23] M. Imran, Y. Ali, Z. Bibi, and M. S. Ghafar, "Characterization of Extremal Unicyclic Graphs Using F-Coindex," Complexity, vol. 2021, Article ID 8480971, 9 pages, 2021.

[24] A. R. Ashrafi, T. Došlić, and A. Hamzeh, "The Zagreb coindices of graph operations," Discrete Applied Mathematics, vol. 158, no. 15, pp. 1571-1578, 2010.

[25] O. C. Havare and A. K. Havare, "Computation of the forgotten topological index and coindex for carbon base nanomaterial," Polycyclic Aromatic Compounds, vol. 2020, pp. 1-13, 2020.

[26] B. Basavanagoud and V. R. Desai, "Forgotten topological index and hyper-Zagreb index of generalized transformation graphs," Bulletin of Mathematical Sciences and Applications, vol. 14, pp. 1-6, 2016.

[27] W. Gao, W. Wang, M. K. Jamil, and M. R. Farahani, "Electron energy studying of molecular structures via forgotten topological index computation," Journal of Chemistry, vol. 2016, Article ID 1053183, 7 pages, 2016.

[28] Y. Bashir, A. Aslam, M. Kamran et al., "Forgotten topological indices of some dendrimers structure," Molecules, vol. 22, no. 6 , p. $867,2017$.

[29] B. Basavanagoud and S. Timmanaikar, "Computing first Zagreb and forgotten indices of certain dominating transformation graphs of Kragujevac trees," Journal of Computer and Mathematical Sciences, vol. 8, no. 3, pp. 50-61, 2017.

[30] S. Akram, M. Javaid, and M. Jamal, "Bounds on F-index of tricyclic graphs with fixed pendant vertices," Open Mathematics, vol. 18, no. 1, pp. 150-161, 2020.

[31] W. Yan, B. Y. Yang, and Y. N. Yeh, "The behavior of Wiener indices and polynomials of graphs under five graph decorations," Applied Mathematics Letters, vol. 20, pp. 290-295, 2007.

[32] M. Eliasi and B. Taeri, "Four new sums of graphs and their Wiener indices," Discrete Applied Mathematics, vol. 157, pp. 794-803, 2009.

[33] H. Deng, D. Sarala, S. K. Ayyaswamy, and S. Balachandran, "The Zagreb indices of four operations on graphs," Applied Mathematics and Computation, vol. 275, pp. 422-431, 2016.
[34] S. Akhter and M. Imran, "The sharp bounds on general sumconnectivity index of four operations on graphs," Journal of Inequalities and Applications, vol. 1, pp. 1-10, 2016.

[35] S. Akhter and M. Imran, "Computing the forgotten topological index of four operations on graphs," AKCE International Journal of Graphs and Combinatorics, vol. 14, no. 1, pp. 70-79, 2017.

[36] M. Javaid, U. Ali, and J. B. Liu, "Computing analysis for first Zagreb connection index and coindex of resultant graphs," Mathematical Problems in Engineering, vol. 2021, Article ID 6019517, 2021.

[37] M. Javaid, S. Javed, S. Q. Memon, and A. M. Alanazi, "Forgotten index of generalized operations on graphs," Journal of Chemistry, vol. 2021, Article ID 9971277, , 2021.

[38] K. Pattabiraman, "F-indices and its coindices of some classes of graphs," Creative Mathematics and Informatics, vol. 26, no. 2, pp. 203-212, 2017.

[39] Z. B. Peng, S. Javed, M. Javaid, and J. B. Liu, "Computing FGZ index of sum graphs under strong product," Journal of Mathematics, vol. 2021, Article ID 6654228, 2021.

[40] Y. Ali, Z. Bibi, and Q. Kiran, "Forgotten coindex of some nontoxic dendrimers structure used in targeted drug delivery," Main Group Metal Chemistry, vol. 44, no. 1, pp. 22-31, 2020.

[41] N. De, S. M. A. Nayeem, and A. Pal, "The F-coindex of some graph operations," SpringerPlus, vol. 5, no. 1, pp. 1-13, 2016. 